\title{
Clarification of Obeticholic Acid Dosing: Response to "Occurrence of Jaundice Following Simultaneous Ursodeoxycholic Acid Cessation and Obeticholic Acid Initiation" by Quigley et al.
}

\author{
Cynthia Feng ${ }^{1}$
}

Received: 6 March 2018 / Accepted: 18 April 2018 / Published online: 10 May 2018

(c) The Author(s) 2018

To the Editor,

I read the case report, "Occurrence of Jaundice Following Simultaneous Ursodeoxycholic Acid Cessation and Obeticholic Acid Initiation," published online on January 5, 2018, by Quigley et al. with interest. I appreciate the authors' communications about the management of primary biliary cholangitis (PBC) with obeticholic acid (OCA); however, as a Medical Director at Intercept Pharmaceuticals, the developers and manufacturers of Ocaliva ${ }^{\circledR}$, I noted some inaccuracies and am writing to ensure that readers have factual and current information as they are considering treatment options for their patients with PBC.

Quotation from Quigley et al.:

Despite the approval of OCA as monotherapy for PBC, clinicians prescribing OCA should ensure that their patient has truly not responded to UDCA before initiating OCA therapy. Moreover, OCA should be introduced as monotherapy only after a UDCA washout period of 3 months has passed to mirror the strategy employed in the other reported OCA trials.

I would like to clarify that Ocaliva ${ }^{\circledR}$ is indicated for the treatment of PBC in combination with ursodeoxycholic acid (UDCA) in adults with an inadequate response to UDCA, or as monotherapy in adults unable to tolerate UDCA [2]. Ocaliva ${ }^{\circledR}$ is not indicated as a monotherapy substitute for UDCA except in the event of UDCA intolerance; it is only in this setting that a 3-month washout was used in the registrational study [3].

Quotation from Quigley et al.:

Cynthia Feng

cynthia.feng@interceptpharma.com

1 Medical Affairs, Intercept Pharmaceuticals, New York, NY, USA
Finally, clinicians should strictly adhere to the recommended dose of up to $10 \mathrm{mg}$ twice a week for their decompensated PBC patients.

I would like to underscore the fact that a dosage of Ocaliva ${ }^{\circledR} 10 \mathrm{mg}$ twice weekly (at least 3 days apart) based on response and tolerability is the maximum dosage for patients with advanced cirrhosis (Child-Pugh Class B or C) or a prior decompensation event. In this patient population, it is important to start with the low dosage of Ocaliva ${ }^{\circledR} 5 \mathrm{mg}$ once weekly. To further clarify, if an adequate reduction in ALP and/or total bilirubin has not been achieved after 3 months of Ocaliva ${ }^{\circledR} 5 \mathrm{mg}$ once weekly, and the patient is tolerating the drug, the recommendation is to increase the dosage of Ocaliva ${ }^{\circledR}$ to $5 \mathrm{mg}$ twice weekly (at least 3 days apart) and subsequently to $10 \mathrm{mg}$ twice weekly (at least 3 days apart) based on response and tolerability.

Patients should continue to be monitored as per standard of care by a healthcare provider with experience managing PBC during treatment with Ocaliva ${ }^{\circledR}$.

In this context, it is also important to mention dosing recommendations for patients who are non-cirrhotic or compensated Child-Pugh Class A. For this patient population, the recommended starting dosage of Ocaliva ${ }^{\circledR}$ is $5 \mathrm{mg}$ once daily in adults who have not achieved an adequate response to an appropriate dosage of UDCA for at least 1 year or are intolerant to UDCA. If an adequate reduction in ALP and/or total bilirubin has not been achieved after 3 months of Ocaliva ${ }^{\circledR}$ $5 \mathrm{mg}$ once daily and the patient is tolerating Ocaliva ${ }^{\circledR}$, the recommendation is to increase dosage to $10 \mathrm{mg}$ once daily, which is the maximum recommended Ocaliva ${ }^{\circledR}$ dosage as per the US prescribing information. The European Medicines Agency (EMA) product information stipulates a recommended starting dose of $5 \mathrm{mg}$ once daily, as well; however, the summary of product characteristics (SmPC) recommends a longer period of assessment of tolerability of 6 months before increasing the dose to $10 \mathrm{mg}$ once daily. 
At Intercept Pharmaceuticals, we want to ensure that patients with PBC get the best possible treatment. For some patients, that may include Ocaliva ${ }^{\circledR}$. When considering Ocaliva ${ }^{\circledR}$, prescribers should be familiar with important safety and dosing information, as well as other information, from the most recent Ocaliva ${ }^{\circledR}$ prescribing information, which can be found at https://ocalivahcp.com.

\section{Reply}

\section{To the Editor,}

We have reviewed Dr. Feng's clarification submitted in response to our recent publication in Digestive Diseases and Sciences regarding the use of Obeticholic acid (OCA) among Primary Biliary Cholangitis (PBC) patients with or without decompensated liver disease [4]. It is indeed important to disseminate this information and keep the physicians abreast of how best to use OCA, particularly in the context of recently released Food and Drug Authority (FDA) reports associating morbidity and mortality among $\mathrm{PBC}$ patients with OCA therapy [5]. However, it remains to be proven whether liver decompensation that occurred among PBC patients was a result of OCA dose, timeline of OCA therapy-either alone or in combination with ursodeoxycholic acid (UDCA) - liver fibrosis or due to the expected progression of liver disease among patients with preexisting liver failure. High-dose OCA monotherapy in PBC patients has not been reported to result in any hepatotoxicity [6]. In contrast, jaundice has been reported among PBC patients with inadequate response to UDCA when high-dose OCA was added to UDCA [7]. Minute details of patients who developed jaundice in association with OCA therapy have not been detailed either in registration trials or FDA reports.

At least in our own anecdotal experience in a patient with compensated cirrhosis, jaundice did not occur due to idiosyncratic or dose dependent OCA toxicity but was found to be possibly related to an abrupt withdrawal of UDCA [4]. Withdrawal of UDCA was not done voluntarily but occurred fortuitously, which unfortunately can occur in doctors' offices where medication errors are not infrequent [8]. Dr. Feng has correctly pointed out that only among those patients who are unable to tolerate UDCA, OCA should be used as monotherapy; and it is in these patients, a 3-month washout period of UDCA is recommended prior to initiating OCA therapy. When the response is inadequate to UDCA, OCA should be added to the therapy, while continuing UDCA therapy. Our case report highlights that stopping
UDCA in a patient responding to it can result in bile acid toxicity, despite being on OCA treatment [4].

Many patients receive a diagnosis of PBC based on laboratory tests only and not necessarily documented by a liver biopsy. Some of these patients may not be correctly diagnosed with cirrhosis; and may develop a subtle transition from compensated to decompensated liver disease, limiting the use of OCA in them, unless very specific follow-up monitoring mechanism is established. Finally, clinicians must adhere to a cautious approach when they use OCA among patients with decompensated liver disease by initiating it at $5 \mathrm{mg} /$ week and gradually increasing up to $10 \mathrm{mg}$ twice weekly dose, based on Intercept Pharmaceutical's recommendations, which were highlighted in our manuscript as well [4].

Gerard Quigley, Abdul Nadir

Department of Gastroenterology, Maricopa Medical Center, Phoenix, AZ, USA

Open Access This article is distributed under the terms of the Creative Commons Attribution-NonCommercial 4.0 International License (http://creativecommons.org/licenses/by-nc/4.0/), which permits any noncommercial use, distribution, and reproduction in any medium, provided you give appropriate credit to the original author(s) and the source, provide a link to the Creative Commons license, and indicate if changes were made.

\section{References}

1. Quigley G, et al. Occurrence of jaundice following simultaneous ursodeoxycholic acid cessation and obeticholic acid initiation. Dig Dis Sci. 2018;63:529-532.

2. OCALIVA (obeticholic acid) [package insert]. Intercept Pharmaceuticals, Inc., New York, NY; 2018.

3. Nevens F, et al. A placebo-controlled trial of obeticholic acid in primary biliary cholangitis. $N$ Engl J Med. 2016;375:631-643.

4. Quigley G, Al Ani M, Nadir A. Occurrence of jaundice following simultaneous ursodeoxycholic acid cessation and obeticholic acid initiation. Dig Dis Sci. 2018;63:529-532.

5. FDA. https://www.fda.gov/Drugs/DrugSafety/ucm576656.htm. 2017.

6. Kowdley KV, Jones D, Luketic V, The OCA PBC Study Group. An international study evaluating the farnesoid $\mathrm{X}$ receptor agonist obeticholic acid as monotherapy in PBC. Journal of Hepatology. 2011;2011:S13.

7. Hirschfield GM, Mason A, Luketic V, et al. Efficacy of obeticholic acid in patients with primary biliary cirrhosis and inadequate response to ursodeoxycholic acid. Gastroenterology. 2015;148:751-761. (e758).

8. La Pietra L, Calligaris L, Molendini L, Quattrin R, Brusaferro S. Medical errors and clinical risk management: state of the art. Acta otorhinolaryngologica Italica. 2005;25:339-346. 